\title{
Influence de la nature de l'azote sur la valeur alimentaire des rations
}

\author{
par H. CALVET et S. DIALLO
}

\begin{abstract}
RESUME
Cette expérimentation, dont le but est de déterminer la façon la plus économique d'enrichir les rations en azote, a porté sur 3 lots de 3 taureaux zébu Gobra soumis à une alimentation iso-énergétique et iso-azotée, les sources d'azote étant de l'urée agricole pour le premier lot, de l'urée alimentaire pour le deuxième et du tourteau expeller pour le troisième.

L'analyse statistique des résultats (poids, hématocrites et urémies) montre que l'urée alimentaire est la source d'azote la plus intéressante, mais cette supériorité ne semble pas liée à des problèmes d'apport azoté, mais à une action de ce produit stimulant la digestibilité des autres composants de la ration.
\end{abstract}

\section{INTRODUCTION}

Depuis plusieurs années sont poursuivis, au Laboratoire National de l'Elevage et de Recherches Vétérinaires, des essais concernant les possibilités d'embouche intensive des bovins tropicaux.

Les diverses expérimentations ont utilisé des rations composées faisant intervenir comme aliment de lest la coque d'arachide mélassée, et des concentrés à base de son et de farine de céréales. Dans chaque cas la ration a dû être enrichie en éléments azotés, et la question s'est posée de savoir quelle était, parmi les produits disponibles sur place, la source d'azote la plus économique.

Le tourteau d'arachide produit en grande quantité au Sénégal, semble d'emblée constituer l'élément idéal de l'enrichissement protéique des rations. Mais son prix (24 F le kg) reste élevé par rapport aux deux autres sources d'azote disponibles, le "Perle urée " et l'urée alimentaire incorporés aux rations à des taux 5 ou 6 fois plus faibles. Le problème de l'azote minéral dans l'alimentation des ruminants et de son efficacité, comparée par rapport aux aliments protéïques, a déjà fait l'objet de plusieurs travaux sans qu'une doctrine définitive ait pu encore être réellement établie.

C'est pour apporter notre contribution à ces recherches et dans un but essentiellement économique que l'expérimentation suivante a été réalisée.

C'est ainsi que trois lots d'animaux comparables ont été soumis pendant trois mois et demi à une alimentation iso-énergétique et isoazotée, alors que dans chaque ration, l'azote adjoint était d'une nature différente.

Dans le lot 1, il s'agissait du "Perle urée ", dans le lot 2 d'urée alimentaire, enfin dans le lot 3 était incorporé du tourteau d'arachide expeller.

\section{MATERIEL ET METHODES}

Le "Perle urée " est l'urée agricole utilisée comme engrais azoté, titrant 47 p. 100 d'azote, contenant certaines impuretés - (et en particulier du bi-urée). Ce produit est vendu à Dakar à $37 \mathrm{~F}$ le kg. L'urée alimentaire est une urée titrant 42 p. 100 d'azote, utilisée pour 
l'alimentation des ruminants, produite en France par l'Office National Industriel de l'Azote. Son originalité réside dans son enrobage et sa présentation en granulés spécialement conditionnés pour faciliter le mélange avec les autres constituants de la ration, et obtenir ainsi une fourniture progressive d'azote à l'animal. Son prix rendu à Dakar est de 42 F le $\mathrm{kg}$.

Enfin le tourteau utilisé provient d'une huilerie de Dakar et titre 49,78 p. 100 de matières protéiques brutes, et il est vendu sur place à $24 \mathrm{~F} \mathrm{le} \mathrm{kg}$.

\section{Les animaux}

Les animaux d'expérience sont constitués par des taureaux zébu de race Gobra âgés de 4 à 5 ans.

Leur poids moyen en début d'expérience est de $230 \pm 4,0$. Ces animaux sont répartis au hasard en trois lots de trois têtes:

- Le lot I reçoit la ration contenant du "Perle urée ".

- Le lot II celle qui contient I'urée alimentaire.

- Le lot III la ration enrichie avec le tourteau d'arachide.

Les animaux sont pesés une fois par semaine et subissent régulièrement chaque semaine trois prises de sang aux horaires suivants: La première se situe à $8 \mathrm{~h}$. avant la distribution de la ration, la deuxième à $11 \mathrm{~h}$. soit $2 \mathrm{~h}$. 30 après la distribution, la troisième à $16 \mathrm{~h}$. une heure trente après la deuxième distribution.

Sur ces prélèvements sont effectués la mesure de l'hématocrite et le dosage de l'urée sanguine.

\section{Les rations}

Les rations sont constituées de 50 p. 100 de coque d'arachide mélassée au taux de 20 p. 100 et 50 p. 100 d'un concentré de composition différente pour chaque lot.

Les formules de ces concentrés sont données ci-après :

\section{Concentré 1 :}

Farine basse de riz . . . . 50

Son de maïs . . . . . . . . . . 40

«Perle urée » . . . . . . . . . 2

C.M.V. (*) , . . . . . . . . 8
Ce concentré titre approximativement $0,86 \mathrm{UF}$ et $25,3 \mathrm{~g}$ d'azote au $\mathrm{kg}$.

\section{Concentré 2 :}

Farine basse de riz . . . . $\quad 50$

Son de maïs . . . . . . . . 40

Urée alimentaire . . . . . . . 2,25

C.M.V. (*) . . . . . . . . . 7,75

Sa valeur est de 0,86 UF et de $25,4 \mathrm{~g}$ d'azote au $\mathrm{kg}$.

\section{Concentré 3:}

Farine basse de riz . . . . 40

Son de maïs . . . . . . . . 40

Tourteau d'arachide . . . . . . 13

C.M.V. (*) . . . . . . . . . 7

Ce qui permet de lui attribuer 0,89 UF et $24,5 \mathrm{~g}$ d'azote au $\mathrm{kg}$.

Ces concentrés sont incorporés dans les mêmes proportions à $50 \mathrm{p} .100$ de coque d'arachide mélassée dont la valeur est estimée à 0,3 UF et $7,31 \mathrm{~g}$ d'azote au $\mathrm{kg}$.

En définitive les quantités d'énergie et d'azote apportées par les rations sont au $\mathrm{kg}$ : Ration $\mathrm{n}^{\mathrm{n}} 1$ : $0,58 \mathrm{UF}$ et $16,3 \mathrm{~g}$ d'azote. Ration $\mathrm{n}^{0} 2: 0,58 \mathrm{UF}$ et $16,3 \mathrm{~g}$ d'azote. Ration $\mathrm{n}^{0} \quad 3: 0,59$ UF et $15,9 \mathrm{~g}$ d'azote.

Ces trois rations sensiblement iso-énergétiques et iso-azotées sont distribuées matin et soir à raison de :

- $4 \mathrm{~kg} \mathrm{du} 18$ avril au 18 mai.

- $5 \mathrm{~kg}$ du 18 mai au 6 juillet.

- $6 \mathrm{~kg} \mathrm{du} 6$ juillet au 10 août.

A partir du 15 juin ont été incorporés des oligo éléments dans les trois rations.

\section{RESULTATS}

Ils portent d'une part sur l'évolution du poids des animaux pendant les 114 jours qu'a duré l'expérience, et d'autre part sur les résultats biochimiques les hématocrites et les urémies.

Il ressort du tableau I qu'il existe à l'intérieur des lots d'importantes variations individuelles. Le gain moyen journalier pour chaque

(*) Complément minéral et vitaminé. 
TABLEAU N"I

Evolution des poids

\begin{tabular}{|c|c|c|c|c|c|c|c|c|c|}
\hline \multirow{2}{*}{$\begin{array}{c}\text { Daces } \\
\text { des } \\
\text { pesées }\end{array}$} & \multicolumn{3}{|c|}{ Lot 1} & \multicolumn{3}{|c|}{ Lot 2} & \multicolumn{3}{|c|}{ Lot 3} \\
\hline & 1 & 2 & 3 & 1 & 2 & 3 & 1 & 2 & 3 \\
\hline 18 avril & 247 & 232 & 227 & 214 & 229 & 229 & 211 & 244 & 226 \\
\hline 27 avril & 227 & 237 & 240 & 220 & $23 B$ & 233 & 214 & 246 & 229 \\
\hline $4 \mathrm{mat}$ & 248 & 243 & 241 & 223 & 242 & 233 & 220 & 244 & 226 \\
\hline 11 mai & 249 & 243 & 237 & 228 & 247 & 240 & 229 & 245 & 223 \\
\hline 18 mal & 256 & 247 & 244 & 239 & 257 & 249 & 233 & 257 & 233 \\
\hline 25 mai & 261 & 252 & 249 & 242 & 261 & 252 & 239 & 261 & 236 \\
\hline 1 juin & 259 & 250 & 248 & 245 & 267 & 252 & 243 & 263 & 235 \\
\hline g Juin & 266 & 256 & 252 & 248 & 267 & 251 & 245 & 266 & 233 \\
\hline 15 juin & 277 & 254 & 267 & 257 & 273 & 261 & 250 & 273 & 246 \\
\hline 22 juin & 282 & 253 & 266 & 266 & 280 & 260 & 252 & 274 & 246 \\
\hline 29 Juin & 288 & 260 & 272 & 267 & 291 & 262 & 260 & 283 & 264 \\
\hline 6 juillet & 290 & 260 & 282 & 275 & 293 & 265 & 266 & 284 & 260 \\
\hline 13 jufllet & 292 & 263 & 282 & 275 & 294 & 276 & 267 & 286 & 263 \\
\hline 20 juillet & 297 & 268 & 283 & 285 & 315 & 274 & 278 & 295 & 267 \\
\hline 27 juillet & 300 & 270 & 290 & 287 & 303 & 277 & 280 & 297 & 270 \\
\hline 3 août & 299 & 276 & 293 & 287 & 320 & 286 & 286 & 316 & 273 \\
\hline 10 août & 320 & 278 & 297 & 290 & 340 & 288 & 286 & 302 & 235 \\
\hline
\end{tabular}

lot (moyenne \pm erreur type de la moyenne) alimentaire, a présenté la meilleure croissance. s'établit ainsi :

$$
\begin{aligned}
& \text { Lot } \mathrm{n}^{\circ} 1: 550 \mathrm{~g} \pm 237 \\
& \text { Lot } \mathrm{n}^{\circ} 2: 713 \mathrm{~g} \pm 425 \\
& \text { Lot } \mathrm{n}^{\circ} 3: 526 \mathrm{~g} \pm 203
\end{aligned}
$$

Au vu de ces résultats, on peut penser que le lot $\mathrm{n}^{\circ} 2$, c'est-à-dire le lot recevant de l'urée

La méthode de calcul suivante doit nous permettre de confirmer cette hypothèse. On établit en effet la droite de régression des poids en fonction du temps pour chaque individu et la comparaison des lots va faire intervenir la pente de chacune de ces droites. Ces pentes sont données dans le tableau suivant.

TABLEAU $N^{\circ} \mathrm{II}$

\begin{tabular}{|l|c|c|c|c|c|c|c|c|}
\hline & \multicolumn{3}{|c|}{ Lot 1 } & \multicolumn{3}{c|}{ Lot 2 } & \multicolumn{3}{c|}{ Lot 3 } \\
\hline $\begin{array}{l}\text { Pentes des } \\
\text { droites de } \\
\text { régression }\end{array}$ & 1 & 2 & 3 & 1 & 2 & 3 & 1 & 2 \\
\hline
\end{tabular}

L'analyse de la variance portant sur ces trois groupes de données, malgré le petit nombre d'animaux et les grosses variations individuelles, fait apparaître une différence significative entre les groupes $(F=6,90)$.
Cette différence est significative entre le groupe 2 comprenant les animaux recevant l'urée alimentaire dans leur ration et l'ensemble des groupes 1 et 3 (Perle urée et tourteau) dont la croissance est comparable. 
On peut donc dire que l'urée alimentaire, introduite dans une ration en tant que complément azoté, rend celle-ci plus efficace que les deux autres sources d'azote que sont le " Perle urée " et le tourteau d'arachide.

Si on se place du point de vue économique, l'enrichissement de l'aliment avec le "Perle urée " revient par $\mathrm{kg}$ de ration à $(37 \times 0,02)$ 0,74 F.CFA, avec l'urée alimentaire $(42 \times$ $0,0225)$ à $0,94 \mathbf{F}$.CFA et $(24 \times 0,13)$ à $3,12 \mathbf{F}$ avec le tourteau.

Seuls le «Perle urée » et liurée alimentaire peuvent donc, du point de vue économique, entrer en compétition mais les 8 p. 100 de bénéfice obtenus sur la croissance avec l'urée alimentaire justifient largement l'utilisation de cette forme d'urée malgré son coût légèrement plus élevé.

\section{RESULTATS BIOCHIMIQUES}

\section{Hématocrite}

L'hématocrite est le résultat de la mesure, après centrifugation, des proportions de globules et de plasma, effectuée sur le sang rendu incoagulable dans un tube gradué très exactement. Il s'agit donc d'un dosage hématologique simple et d'une assez grande précision dont on serait en droit d'attendre des enseignements intéressants.

Malheureusement, la variabilité de l'hématocrite est très grande et a fait, chez l'animal tout au moins, l'objet d'études peu nombreuses.

En pathologie, la diminution de l'hématocrite est nette au cours des diverses anémies. L'hématocrite est également perturbé dans les rétensions hydriques dues à des cardiopathies dans les déshydratations par perte de sel ou des plasmorragies (brûlures étendues) où le volume plasmatique diminue de façon sensible alors que le volume globulaire reste normal.

En physiologie, l'hématocrite constitue une donnée d'interprétation encore plus complexe.

Les travaux de CHARPENTIER et de BONHOMME sur l'hématocrite des veaux et des femelles adultes apportent cependant quelques lumières dans ce domaine. Ces auteurs ont étudié la variabilité de l'hématocrite sur une population de 1.276 vaches de plusieurs races françaises et ont déterminé la part qui, dans la variation totale, tient à la région, à l'exploitation et à la variabilité individuelle intra exploitation. Ces proportions sont respectivement les suivantes: 13,1 p. $100,14,7$ p. 100 et 72 p. 100.

L'hématocrite tient donc pour la plus grande partie à des facteurs individuels et génétiques qui seraient susceptibles de se transmettre dans la descendance. Ces mêmes auteurs ont, en effet, établi une corrélation entre la valeur de l'hématocrite de la mère et celui de son veau à 8 jours. $(r=+0,52)$.

Le milieu, et, essentiellement, les facteurs nutritionnels agiraient pour une part beaucoup plus faible que les auteurs estiment à 27,8 p. 100 .

Au cours de l'expérience rapportée dans cette note, les hématocrites des 9 animaux d'expérience sont dosés une fois par semaine au cours des trois prélèvements effectués le même jour.

L'un se situe à 8 h. 30 sur les animaux à jeûn, l'autre à $11 \mathrm{~h}$. soit $2 \mathrm{~h}$. 30 après la première distribution d'aliment et le troisième à $16 \mathrm{~h}$. soit $1 \mathrm{~h}, 30$ après la deuxième distribution.

Les résultats généraux sont rapportés dans le tableau suivant:

TABLEAU N ${ }^{\circ}$ III

Evolution des hêmatocrites en fonction du temps, après le repas.

\begin{tabular}{|c|c|c|c|c|c|c|c|c|c|}
\hline \multirow{2}{*}{ Horaire } & \multicolumn{3}{|c|}{ Lot 1} & \multicolumn{3}{|c|}{ Lot 2} & \multicolumn{3}{|c|}{ Lot 3} \\
\hline & 1 & 2 & 3 & 1 & 2 & 3 & 1 & 2 & 3 \\
\hline $8 h 30$ & $36 \quad \pm 0,83$ & $36,6+2,67$ & $43 \pm 0,76$ & $37 \pm 1,0$ & $37,7 \pm 0,7$ & $31,4 \pm 2,2$ & $30,2 \pm 0,96$ & $31,6 \pm 0,63$ & $31 \pm 1,73$ \\
\hline $11 \mathrm{~h}$ & $36,2 \pm 1,11$ & $32,5+2,02$ & $38,7 \pm 0,76$ & $34,5 \pm 1,20$ & $35,4 \pm 0,71$ & $29,6 \pm 1,54$ & $29,3 \pm 0,75$ & $30,1 \pm 0,63$ & $30,2 \pm 1,45$ \\
\hline $16 \mathrm{~h}$ & $36,3 \pm 0,93$ & $35,2 \pm 1,98$ & $38,6 \pm 1,36$ & $36,4 \pm 1,05$ & $35,7 \pm 0,72$ & $31 \pm 2,30$ & $28,9+4,12$ & $37,7 \pm 1,12$ & $29,7 \pm 1,4$ \\
\hline
\end{tabular}


A l'examen de ce tableau on constate :

1. Que la variabilité des données pour chaque individu est d'une façon générale importante, et n'est pas différente d'un prélèvement à l'autre. Donc, aucun des trois ho- raires de prélèvement ne paraît plus favorable pour le dosage de l'hématocrite.

2. Si on décompose la variation totale entre les diverses sources de variation, on obtient le tableau suivant: (Pour les prélèvements de 8 h. 30)

TABLEAU $\mathbb{N}^{*} \mathrm{IV}$

\begin{tabular}{|c|c|c|c|c|}
\hline Sources de variation & Sonme des carrês & $\mathrm{DL}$ & Carré moyen & $\mathrm{F}$ \\
\hline Variation totale & 16.644 & 401 & & \\
\hline $\begin{array}{l}\text { Variation liée aux diffērences } \\
\text { individuelles }\end{array}$ & 4.009 & 8 & 501 & $20++$ \\
\hline Variatıon liée aux rêgímes & 3.101 & 2 & 1.550 & $64++$ \\
\hline Varlation liêe aux horalres & 237 & 2 & 118 & $4,9+$ \\
\hline Variation résiduelle & 9.297 & 389 & 24 & \\
\hline
\end{tabular}

Il ressort de ce tableau les conséquences suivantes :

1. La composition des rations a eu une influence hautement significative sur les valeurs de l'hématocrite.

Les animaux recevant la ration avec le "Perle urée " ont l'hématocrite le plus élevé, l'hématocrite le plus bas se rencontrant avec la ration au tourteau d'arachide. Il est possible que cette différence tienne à une régulation du métabolisme hydrique. Le "Perle urée ", ayant probablement la faculté de stimuler la diurèse, entraînerait un certain degré d'hémo-concentration.

2. Les différences liées aux individus sont également hautement significatives. L'hématocrite constitue donc pour une grande part une sorte de constante individuelle qui pourrait même pour CHARPENTIER et collab. être capable d'une certaine héritabilité.

3. Les hématocrites diffèrent significativement aux trois horaires de prélèvement. Le plus élevé est celui de $8 \mathrm{~h}$. 30 et le plus bas celui de 11 h. A 11 h. en effet, après l'administration du repas, les animaux se trouvent en pleine digestion et le plasma véhicule les nutriments absorbés. Son volume relatif est alors augmenté ce qui se traduit par une diminution de l'hématocrite. La différence entre les hématocrites de 8 h. 30 et $11 \mathrm{~h}$. pourrait donc constituer une sorte de critère de valeur de la ration. Dans notre expérimentation cette différence est maximale pour la ration contenant l'urée alimentaire.

\section{Urémie}

Comme pour l'hématocrite, l'urée sanguine a été dosée sur chaque animal une fois par semaine au cours de trois prélèvements effectués à 8 h. $30,11 \mathrm{~h}$. et $16 \mathrm{~h}$.

La méthode de dosage intervient au premier chef dans la détermination de l'urée sanguine comme l'a souligné LABOUCHE dans son travail sur l'urée des ruminants. La méthode utilisée au cours de cette recherche a été celle du "micro dosage de l'urée par la méthode enzymatique ", au cours de laquelle l'urée est transformée quantitativement en ammoniac par l'uréase et l'ammoniac dosé colorimètriquement par le réactif de NESSLER. Les résultats de ces dosages sont présentés dans le tableau V.

L'étude de ces données a été entreprise dans le cas précédent par une analyse de variance hiérarchisée au cours de laquelle ont été identifiées les diverses sources de variation : variation individuelle, variation liée au régime, variation tenant aux horaires de prélèvement. Les calculs permettent l'établissement du tableau VI.

Il ressort de ce tableau que l'urémie des animaux en expérience a varié de façon significative d'un prélèvement à l'autre au cours de 
TABLEAU $\mathrm{N}^{\circ} \mathrm{V}$

Evolution de 1'urêe sangulne en fonction du temps, après le repas.

\begin{tabular}{|c|c|c|c|c|c|c|c|c|c|}
\hline \multirow{2}{*}{ Horaire } & \multicolumn{3}{|c|}{ Lot I } & \multicolumn{3}{|c|}{ Lot 2} & \multicolumn{2}{c|}{ Lot 3 } \\
\cline { 2 - 10 } $3 \mathrm{~B} 30$ & $382 \pm 24$ & $391 \pm 27$ & $398 \pm 26$ & $360 \pm 29$ & $377 \pm 24$ & $412 \pm 23$ & $353 \pm 25$ & $387 \pm 29$ & $367 \pm 29$ \\
\hline $11 \mathrm{~b}$ & $442 \pm 24$ & $412 \pm 47$ & $471 \pm 17$ & $447 \pm 24$ & $427 \pm 24$ & $470 \pm 17$ & $403 \pm 33$ & $403 \pm 23$ & $405 \pm 30$ \\
\hline $16 \mathrm{~h}$ & $425 \pm 25$ & $404 \pm 38$ & $419 \pm 19$ & $364 \pm 18$ & $409 \pm 26$ & $416 \pm 37$ & $369 \pm 30$ & $324 \pm 23$ & $306 \pm 23$ \\
\hline
\end{tabular}

TABLEAU N"VI

\begin{tabular}{|c|c|c|c|c|}
\hline Sources de varfation & Somme des carrés & $\mathrm{DL}$ & Carrë moyen & F \\
\hline Variation totale & 5.299 .354 & 404 & & \\
\hline $\begin{array}{l}\text { Vartation liée aux différences } \\
\text { individuelles }\end{array}$ & 253.816 & 8 & 31.727 & $2,69++$ \\
\hline Varlation liẻe aux réglmes & 162.478 & 2 & 81.239 & $6,90++$ \\
\hline Varlation liēe aux horatres & 272.781 & 2 & 136.390 & $11,59++$ \\
\hline Variation résiduelle & 4.610 .279 & 392 & 11.760 & \\
\hline
\end{tabular}

la même journée. Parmi les horaires retenus, c'est à 11 heures que le taux d'urée est le plus élevé, soit 2 heures et demi après le repas et au moment où l'ammoniogénèse dans le rumen est la plus élevée, comme l'ont montré les expérimentations sur la biochimie du rumen réalisées dans la même section.

Ce fait souligne l'absolue nécessité, lorsqu'on veut obtenir des données sur l'urémie des ruminants, d'effectuer des prélèvements à des horaires stricts établis en fonction de l'heure à laquelle est administré le repas.

La variation individuelle est très importante. Dans les observations effectuées sur les urémies du troupeau de Sangalkam par LABOUCHE, cet auteur avait déjà noté des coefficients de variation s'échelonnant suivant les mois de 11,7 à 29,2 p. 100.

Enfin, dans cette expérimentation, les trois rations ont entraîné une variation hautement significative de l'urémie. La somme des $\mathrm{X}$ pour les trois groupes d'animaux est la suivante: Ration «Perle urée »: SX . . 55.727 Urée alimentaire : SX . . . . . 55.302 Tourteau d'arachide : SX . . . 49.790
Les deux rations de l'urée ont l'urémie la plus élevée, celle à l'urée alimentaire semblant plus basse. Le tourteau d'arachide entraîne des données inférieures, ce qui tendrait à prouver que, dans le rumen, l'azote protéique du tourteau n'est attaqué que partiellement pour donner de l'ammoniac, tandis qu'une part plus ou moins importante passerait dans l'intestin et serait alors absorbée sous forme d'acides aminés. Ceci semblerait un gage d'une meilleure utilisation de l'azote qui, dans le cas présent, ne se traduit pas par un bénéfice pour l'animal puisque c'est avec la ration au tourteau que les gains de poids sont les plus faibles. On peut donc penser que la supériorité des rations contenant de l'urée n'est pas liée à des problèmes d'azote. Sans doute l'urée, particulièrement sous sa forme d'urée alimentaire, a agi, comme d'autres auteurs l'ont déjà souligné, en stimulant la flore ruminale et en provocant une meilleure digestibilité des constituants de la ration et en particulier de la cellulose.

\section{CONCLUSIONS}

Le but de cette expérimentation était de déterminer la nature de l'azote qu'il convenait 
d'incorporer aux rations composées pour en obtenir le meilleur rendement économique.

Parmi les trois rations iso-énergétiques et iso-azotées expérimentées, c'est celle qui contient de l'urée alimentaire qui entraîne les meil- leurs gains de poids. La valeur supérieure de cette dernière ration ne semble pas être liée à des problèmes d'apport azoté mais à une action de ce produit stimulant la digestibilité des autres composants.

\section{SUMMARY}

\section{Influence of the nitrogen origin on the nutritive value of fattening diets}

This experiment was aimed to determine the most economical way to increase the nitrogen content of diets used to fatten Zebu cattle. It was carried out on 3 batches of Zebu Gobra bulls fed with diets presenting the same rate of nitrogen (iso nitrogenous), the difference bearing on the nitrogen sources. The first batch received agricultural urea, the second alimentary urea and the third one ground-nut cake.

The statistical analysis of the results (weights, haematocrites, and uraemias) shows that alimentary urea is the nitrogen source the most interesting. It was found that this superiority does not seem to be in connection with problems of nitrogenous supply but that alimentary urea exerts a stimulating action on digestibility of other components of the diet.

\section{RESUMEN}

Influencia de la natura del nilrógeno sobre el valor alimenticio de las raciones

Con el objeto de determinar el modo más económico de enriquecer las raciones con nitrógeno, se hizó una experimentación sobre 3 lotes de 3 toros cebú Gobra alimentados por raciones iso-energéticas e isonitrogenadas, siendo la origen del nitrógeno la urea agricola para el primer lote, la urea alimenticia para el segundo y la torta expeller para el tercero.

El análisis estadístico de los resultados (peso, hematocritos y uremias) muestra que la urea alimenticia es la origen de nitrógeno más interesante, pero esta superioridad no parece ligada con problemas de traida nitrogenada sino con una acción de dicho producto estimulando la digestibilidad de los demás ingredientes de la ración.

\section{BIBLIOGRAPHIE}

1. CHARPENTIER (J.), BONHOMME (D.), «Facteurs de variation de l'hématocrite des bovins. II. Hématocrite des vaches adultes", Ann. Zootech., 1968, 17 (3): 327-35.

2. LABOUCHE (C.), «Contribution à la connaissance du transit de l'urée chez les ruminants. Recherches sur l'urémie et l'élimination rénale de l'urée chez les bovins domestiques en milieu tropical », Thèse. Sciences. Toulouse. 1967. no 306.

3. LOOSLI (J.K.), McDONALD (I.W.), « Azote non protéique dans l'alimentation des ruminants", Rome, F.A.O., 1968. (Coll. Etudes agricoles de la F.A.O., n० 75).

4. BRIGGS (M.H.), ed.. «Urea as a protein supplement », Oxford, Pergamon Press, 1967. 